Aus dem Allgemeinen Krankenhause St. Georg in Hamburg. Abteilung für Haut- und Geschlechtskrankheiten. (Dirigierender Arzt: Oberarzt Dr. Ed. Arning.)

\title{
Zur Kenntnis der Keratosis follicularis Morrow-Brooke.
}

Von

Dr. Felix Lewandowsky (Hamburg).

Häufiger wohl als irgend eine andere Hautklinik Deutschlands bietet die Dermatologische Abteilung des Allgemeinen Krankenhauses Hamburg St. Georg Gelegenheit, Angehörige fremder Rassen zu untersuchen. Meist sind es auf den Schiffen als Heizer dienende Indier, Neger und Chinesen, die nach der Ankunft im Hafen wegen einer unterwegs aquirierten Geschlechtskrankheit ins Krankenhaus eingeliefert werden. Bei der Untersuchung lassen sich zuweilen ganz interessante Nebenbefunde erheben. Um einen solchen handelt es sich in dem hier mitzuteilenden Falle, der einen jungen Indier betraf. Die Veröffentlichung scheint mir berechtigt, weil das betreffende Krankheitsbild schon in Europa außerordentlich selten, bei einem Individuum farbiger Rasse aber, soviel ich sehe, bisher noch nicht beobachtet worden ist.

Anamnese: Uozza Ali, 25jähriger Indier, Heizer, wurde am 25./II. 1909 wegen eines Ulcus molle ins Krankenhaus geschickt. Bei der Aufnahme wurde eine eigentümliche, fast über den ganzen Körper verbreitete Hautaffektion festgestellt. Da Patient kein Wort einer europäischen Sprache spricht, so ist mit ihm nur auf pantomimische Art eine Verständigung möglich.

Als er unser Interesse an seiner Hantkrankheit bemerkt, scheint er mit einer ziemlich ausdrucksvollen Gebärdensprache folgendes sagen zu wollen: die eigentümliche Hautbeschaffenheit habe nichts zu bedeuten, er habe sie schon als ganz kleines Kind gehabt. Ebenso verneint er die Frage, ob ihm das Leiden Jucken verursache. Auch im weiteren Verlaufe wurde niemals beobachtet, daß er sich kratzte. Irgend welche genaueren An- 
gaben wie über Heredität u. dgl. waren natürlich nicht herauszubekommen.

Status praesens: Schlank gewachsener, kräftiger Mann. Innere Organe normal. Kleines Ulcus molle im Sulcus coronarius. Keine Drüsenschwellungen.

Die Haut des Patienten zeigt eine merkwürdige, symmetrisch ausgebreitete Affektion. Am stärksten befallen sind die Extremitäten und hier wieder die Streckseiten, besonders der Vorderarme. Andere Prädilektionstellen sind die Knie, die Glutaealgegend, die Rückenflächen der ersten Fing erphalangen. Ergriffen sind ferner Wangen und Ohren, in weit geringerem Grade der Hals und die Seitenteile des Rumpfes. Vollkommen frei sind der behaarte Kopf, die Genitalien, Inguinalgegend, Handfächen, Fußsohlen und Gelenkbeugen. Die Dermatose besteht aus zahlreichen papulösen, stark prominierenden Einzeleffloreszenzen von Stecknadelkopf- bis Hanfkorngröße. Die Farbe derselben ist teils braun wie die der normalen Haut des Patienten, teils tief dunkelbraun bis schwarz; einige wenige lassen am Rand einen schwach rötlichen Farbenton erkennen. Das Zentrum einer jeden Papel wird von einer derben dunkel gefärbten Hornmasse gebildet. Diese ragt entweder als barter spitzer Stacbel 1 bis $3 \mathrm{~mm}$ über die Oberfläche hervor, oder sie hat das Aussehen eines komedoartigen Propfes. Durch starken seitlichen Druck auf die Papeln kann man bei einzelnen den Hornpfropf ausquetschen, der von trockner harter Konstistenz ist; im Zentrum bleibt an seiner Stelle eine Vertiefung. Die Anordnung der Effloreszenzen ist je nach der Lokalisation verschieden. Bald stehen sie so dicht gedrängt, daß sie hie und da zu kleinen Verrukositäten vereinigt sind, wie an den Streckseiten der Vorderarme; bald stehen sie ganz isoliert, wie an den Beugeflächen. Anf den Fingerrücken bilden sie regelmäßige kleine Hornkegel, an den Ohrrändern dicht nebeneinanderstehende Stacheln, auf den Wangen mehr unregelmäßig geformte Hornpfröpfe. Eine wirkliche Gruppenbildung ist nirgends zu erkennen. An Nacken und Kinn finden sich nur einzelne kJeine Effloreszenzen, ebenso an den Seitenpartien des Rumpfes. Hier treten die Follikel etwas stärker als normal hervor, der eine oder andere trägt eine feine hornige Spitze. Auch sonst scheinen makroskopisch die einzelnen Läsionen den Haarfollikeln zu entsprechen.

Die Haut zwischen den einzelnen Papeln scheint nicht pathologiseh verändert. Eibenso fühlt sich die Haut dort, wo keine Effloreszenzen stehen, wie an den mittleren Rumpfpartien vollkommen glatt an und gibt das bei dieser Rasse eigentümliche weiche Gefühl beim Darüberstreichen, das sich aus dem Fehlen jeder stärkeren Körperbehaarung erklärt.

An den Mundwinkeln zeigt sich eine abnorme Verhornung der Wangenschleimhaut. Es finden sich hier dicke weibliche von Spalten. durchfurchte Auflagerungen, wie bei einer hochgradigen Leukoplakie. Die Zunge ist nicht verändert.

Nägel, Handflächen und Fußsohlen sind völlig normal. 
Histologische Untersuchung: Es wurden zwei kleine Hautstücke rom Vorderarme exzidiert in Alkohol und in Sublimateisessig fixiert, in Paraffin eingebettet und in Serien geschnitten. Gefärbt wurde mit Hämalaun-Eosin, Eisenhämatoxylin - van Gies on (diese Färbung gab die schönsten Bilder), Kresylechtviolett, Pikrokarmin und nach Gram.

Als Hautveränderung tritt schon bei oberfächlicher Betrachtung eine starke Hyperkeratose der Follikel hervor. Die Verhornungsanomalie hat teils den ganzen Follikel ergriffen, teils nur den Follikelausgang. Im ersteren Falle ist der Haarbalg trichterförmig erweitert, angefüllt mit parallel zur Wand verlaufenden Hornlamellen, in deren Mitte sich meist ein grade verlaulendes Lanugohaar findet, dessen Kolben unten in der Spitze des Follikeltrichters wurzelt. Außer dieser einfachsten Form finden sich Follikel, die zwar in toto verhornt sind aber an ihrem Hals stärker als in den tieferen Partien. Diese Follikel beginnen an der Haarwurzel sich trichterförmig zu erweitern, dehnen sich aber am Halse plötzlich ganz bedeutend aus. Bei anderen ist der Follikelhals von mächtigen Hornmassen ausgefüllt und erweitert, während der normale tiefere Follikelteil nur als ein kleiner Appendix erscheint. Die größten weit über das Hautniveau emporragenden Hornmassen fanden sich an Stellen, wo zwei Follikel in einen stark erweiterten Hals münden. Hier verlaufen die Haare spiralig innerhalb des Hornstachels. Wieder andere Stachel werden durch Hornlamellen gebildet, die im Follikelhals wie flache Schalen aufeinander gehäuft sind. Schließlich sind auch noch Retentionserscheinungen $\mathrm{zu}$ beobachten. $\mathrm{Ob}$ die Hornschicht in toto verdickt ist, darüber kann ich ein sicheres Urteil nicht abgeben. Verglichen mit einem Hautstück vom Unterschenkel eines anderen Indiers (abheilende syphilitische Papel und normaler Rand) scheint allerdings die Hornschicht in unserm Falle etwas breiter und kompakter zu sein, doch nicht sehr beträchtlich; größer ist der Unterschied schon im Vergleich mit der Haut eines Europäers, doch gehört wohl eine stärkere Hornschicht zu den Eigentümlichkeiten der Haut jener Rasse. Sicher konnte. dagegen in der Umgebung vieler Follikel eine nicht unbedeutende Verdickung der Hornschicht konstatiert werden. Diese 
verdickte Hornschicht zieht nun an manchen Stellen über die Follikel hinweg und verwehrt dadurch den intrafollikulären Hornmassen den Austritt. Infolgedessen sind diese Follikel nach Art eines seborrhoicshen Kokons oder sackartig erweitert, am Halse aber verengt. Nur im Zentrum scheint eine Art Perforationsöffnung zu bestehen.

Die Hornmassen zeigen im allgemeinen die Charaktere reiner Hyperkeratose, nur im Zentrum einzelner Stacheln finden sich auch parakeratotische Herde. Die Keratohyalinschicht ist an manchen Follikeleingängen etwas verbreitet, an den Seitenwänden der hyperkeratotischen Follikel meist nur von zwei Lagen ganz platt gedruckter Zellen gebildet; in der Tiefe werden diese Zellen wieder breiter, das Keratobyalin in den einzelnen aber spärlicher. Das Rete ist an den Seitenwänden der verhornten Follikel häufig atrophisch, auf wenige Zellagen beschränkt, im übrigen aber von normaler Breite.

Besondere Aufmerksamkeit verdient die atypische Verhornung des Follikelepithels unter mehr oder weniger reichlichem Auftreten jener Gebilde, die Darier bei der nach ihm benannten Dermatose zuerst als "Corps ronds" und "Grains" beschrieben hat. Diese sind über die ganze Wand des befallenen Follikels verteilt und finden sich nicht bloß in unmittelbarer Nachbarschaft des Hornzapfens sondern auch nahe der Basalschicht. Man kann alle Übergänge von der normalen Epithelzelle zu jenen Formen beobachten, besonders schön bei Eisenhämatoxylin-van Gi es o n-Färbung. Im ersten Stadium unterscheiden sie sich von den anderen Zellen nur durch die große kreisrunde Gestalt und das Fehlen der Interzellularbrücken; oft ist schon eine die Zelle einschließende Membran und eine helle Zone um den Kern angedeutet. In der weiteren. Entwicklung nimmt das Protoplasma einen leuchtend rötlichgelben Farbenton an, der Kern wird kleiner und tiefer gefärbt um ihn herum bildet sich eine helle Zone und auf diese folgt nach außen eine Zone, in der Keratohyalinkörnchen liegen. Viele Zellen sind von einer deutlichen Membran eingeschlossen; zuweilen umgibt eine Membran zwei oder drei Gebilde. Schließlich rerschwindet der Kern; an seiner Stelle sind manchmal noch einzelne Granula zu erkennen, die Zelle verwandelt sich 
in ein rundes oder orales stark glänzendes gelbes Körperchen, die "Grains". Dariers. Diese letzteren finden sich reichlich auch in den tieferen Partien des Hornpfropfes, zwischen den normalen Hornlamellen. Zuweilen sieht man ein verhorntes Gebilde innerhalb einer Epithelzelle; bei Pikrokarminfärbung stellt sich das folgendermaßen dar: ein gelber kreisrunder Körper im Zentrum einer Zelle, diesem an einer Seite anliegend der rotgefärbte, platt gedruckte Zellkern, nach außen das hellrosa gefärbte Protoplasma, das ganze von einer Membran umgeben.

Eine Vermehrung des Pigmentes wurde nirgends bemerkt. Das Pigment findet sich beim Indier über sämtliche Schichten des Rete Malpighi verteilt; aber auch die Hornschicht enthält reichlich Pigmentkörnchen. Die epitheliale Wand der verhornten Follikel enthielt eher weniger Pigment als die Umgebung. Die dunkle Färbung vieler Effloreszenzen ist also woh1 auf die starke Verdickung der pigmenthaltigen Hornschicht in der Umgebung der erkrankten Follikel zurückzuführen.

Eine Talgdrüse wurde an keinem Follikel gefunden. Das Rete Malpighi außerhalb der Follikel war normal. Die Kutis zeigt keine Veränderungen außer an einzelnen Stellen nicht ganz unerheblichen perivaskulären Lymphozyteninfiltraten um die befallenen Follikel herum.

Verlauf: Das Ulcus molle heilte in wenigen Tagen auf lokale Behandlung. Da bei dem Patienten aber die Wassermann sche Serumreaktion auf Lues positiv ausgefallen war, so wurde er einer Schmierkur unterworfen. Als diese nach 4 Wochen beendet war, konnte eine wesentliche Besseruvg der Verhornungsanomalie, besonders an den mit der Salbe direkt in Berührung gekommenen Körperteilen konstatiert werden. Doch konnte von einer Heilung der Affektion nicht die Rede sein. Besonders die Fingerrücken blieben unverändert und auch die Glutaealgegend war noch stark befallen. Der Patient wurde in diesem gebesserten $\mathrm{Zu}$ stand in die Heimat entlassen.

Diagnostiziert wurde die Affektion als Keratosis follicularis (Morrow-Brooke). Um diese Diagnose zu begründen, müssen wir zuerst den Verdacht ausschließen, daß es sich um Erscheinungen von Syphilis gehandelt habe. Das bei Lues Hyperkeratosen vorkommen können, ist ja bekannt, und wir hatten erst ror kurzem Gelegenheit ebenfalls auf der Abteilung von Herrn Arning einen Fall zu beobachten, 
in dem die Krankheit - wenn man so sagen darf - das Hornsystem in eigentümlicher Weise befallen hatte. Bei einem Patienten mit sekundärer Lues zeigten sich neben einem über den Rumpf verbreiteten makulösen Exanthem an Nacken und Streckseiten der Vorderarme einzelne Gruppen stark prominierender schwach rötlicher papulöser Effloreszenzen, die den Follikeln zu entsprechen schienen, von denen jede im Zentrum einen derben Hornstachel trug. Außerdem fanden sich dicke schwielenartige Auflagerungen an Handfächen und Fußsohlen, um sie herum ein leicht geröteter Hof. Sämtliche Finger und Zehennägel waren hochgradig verdickt, schwärzlich gefärbt von höckeriger Oberfläche und brüchig, Nagelfalz und -bett entzündet. In den verhornten Papeln am Nacken und unter den Schwielen wurden Spirochaetae pallidae nachgewiesen. Auf spezifische Behandlung heilten alle diese Erscheinungen. Histologisch fand sich eine Hyperkeratose der Follikelausgänge bei normaler Beschaffenheit des unteren Follikelteiles und gut erhaltenen Talgdrüsen, Ödem des Papillarkörpers in der Umgebung und Infiltration mit Rund- und Epitheloidzellen. Über den Papillenspitzen war das Epithel teilweise gelockert von reichlichen parakeratotischen Hornlamellen bedeckt; an einzelnen Stellen war es auch durch Austritt von Serum und Leukozyten zur Bildung kleiner Krusten gekommen.

Unser Fall war aber von dem eben erwähnten klinisch und histologisch doch recht verschieden. Es war bei ihm kein Symptom von Lues nachzuweisen. Mit der Wassermannschen Reaktion hat es hier seine eigene Bewandnis. Herr Reinhart, der auf der Arningschen Abteilung bei einer großen Anzahl luetischer und nicht luetischer Patienten die Serumreaktion angestellt hat, hat bei den Indiern eine merkwürdige Beobachtung gemacht.

Er hat im ganzen 27 Indier untersucht. 13 von ihnen waren syphilitisch erkrankt; diese reagierten positiv bis auf zwei, die sich im Primärstadium vor Auftreten sekundärer Erscheinungen befanden. Ein Patient, der sonst keine syphilitische Symptome zeigte, hatte eine Perforation des Gaumens, muß also als verdächtig bezeichnet werden; auch dieser reagierte positiv. Bei den 13 übrigen indischen Patienten lag klinisch kein 
Verdacht auf Lues vor; trotzdem gaben sie alle bis auf einen eine positive Serumreaktion. Dieses Ergebnis ist um so auffallender, als Herr Reinhart bei seinen übrigen Untersuchungen, die er kürzlich publiziert hat (Münch. med. Woch. 1909) durchaus die günstigen Angaben über den diagnostischen Wert der Reaktion bestätigt hat. Eine Erklärung der Tatsache ist einstweilen noch nicht möglich. Muß man bei all diesen Leuten eine latente Lues annehmen oder gibt es in ihrer Heimat andere Krankheiten, die das Serum dauernd im Sinne jener Reaktion verändern? Wie dem auch sei, sicher kann der positive Ausfall der Reaktion in unserem Falle uns nicht von der syphilitischen Natur der Affektion überzeugen.

Ferner glauben wir den Patienten richtig verstanden zu haben, daß sein Leiden von Kindheit an bestehe. Schließlich brachte eine $\mathrm{Hg}$-Einreibungskur keine Heilung. Daß eine Besserung eintrat ist nicht weiter wunderbar, denn erstens ist die Keratosis follicularis großen spontanen Schwankungen in der Intensität unterworfen, zweitens sind auf indifferente Salbenbehandlung Besserungen beschrieben und schließlich empfiehlt z. B. Thibierge speziell Quecksilbersalben bei der unserer Affektion außerordentlich nahestehenden, wenn nicht mit ihr identischen „Acné cornée".

Wenn wir somit glauben, Syphilis ausschließen zu können, so bringt es die von uns gestellte Diagnose „Keratos is follicularis Morrow-Brooke" mit sich, daß wir noch zwei Krankbeitsbilder in die Diskussion ziehen müssen, den Lichen spinulosus und die Dariersche Krankheit.

Zum Lichen spinulosus bestehen von zwei Seiten her Beziehungen mit der Brookeschen Keratosis. Denn einerseits hat Brooke selber in seiner grundlegenden Publikation den Namen Lichen spinulosus als ein Synonym für die von $\grave{i h m}$ beschriebene Affektion erwähnt, wohl mit Unrecht. Anderseits aber haben die französischen Dermatologen unter dem Namen A c né cornée eine ganze Anzahl unter sich rerschiedener Beobachtungen veröffentlicht, von denen die einen für den Lichen spinolosus, die andern für die Keratosis follicularis Brooke von neueren Autoren in Anspruch genommen sind. Ich habe vor fünf Jahren gelegentlich eines Falles aus der Jadas. 
sohnschen Kinik in Bern den einen Teil dieser Frage bereits behandelt. Damals war ich außer der Beschreibung Crockers nur auf einzelne kasuistische Mitteilungen angewiesen. Seitdem aber sind drei große zusammenfassende Arbeiten über Lichen spinulosus erschienen, von Adamson in London, von Salinier aus der Audryschen Klinik in Toulouse und von Piccardi in Turin. In allen droi Arbeiten ist das gesamte Material sorgfältig zusammengetragen und gesichtet. Adams on kommt dabei zu dem Resultat, daß mein Fall kein Lichen spinulosus sei, wegen der ausgesprochen entzündlichen Erscheinungen im Anfang; auch Salinier und ihm folgend Pic cardi meint, dal hier kein eigentlicher Lichen spinulosus sondern ein "sekundärer Spinulosismus" im Verlaufe einer Infektionskrankheit vorliege. Wenn ich auch die Abweichung meines Falles ron dem typischen Bild anerkenne, so kann ich doch diese Erklärung Saliniers nicht ohne weiteres annehmen. Denn über einen solchen sekundären Spinulosismus bei akuten Hautinfektionen ist doch bisher nichts bekannt. Anders ist es bei Lues, wofür ich oben selbst ein Beispiel aus der A rningschen Abteilung angeführt habe, und bei Tuberkulose, d. $h$. beim Lichen scrofulosorum. Ich habe noch in der Jadas s ohnschen Klinik einen Fall von Lichen scrofulosorum bei einem ichthyotischen Kinde gesehen, bei dem jede Effloreszenz in der Mitte einen kleinen Hornstachel trug, (Observation III in der Arbeit von Lesseliers). Aber bei akuten Follikulitiden um solche konnte es sich ja in meinem Falle nur handeln -, welche doch zu den häufigsten Hauterkrankungen gehören, habe ich etwas ähnliches nie gesehen und auch in der Literatur nichts derartiges gefunden. Während meiner Anwesenheit in London im vorigen Jahre hatte Herr Adams on die Freundlichkeit, mir einen Fall von Lichen spinulosus zu zeigen Abgesehen von der viel geringeren Ausdehnung der Affektion in diesem Falle, bestand, was den Einzelherd anbetrifft, größte Ähnlichkeit mit dem zweiten Stadium des von mir beschriebenen Falles. Von der Acné cornée der Franzosen reiht A da mson eine ganze Anzahl Fälle in den Lichen spinulosus ein, während Salinier einer solchen Identifizierung widerspricht. Ich möchte mich hierin Salinier anschließen und glaube, daß 
die meisten Beobachtungen mehr Gemeinsames mit der B rookeschen Dermatose haben als mit dem Lichen spinulosus.

Heute, wo durch verschiedene Arbeiten aus den letzten Jahren beide Krankheitsbilder schärfer umgrenzt worden sind, kann es eigentlich keine Schwierigkeiten bereiten die Keratosis follicularis Morrow-Brooke vom Lichen spinulosus zu unterscheiden, den wir mit Salinier vielleicht besser als Keratosis spinulosa bezeichnen. Die letztere Krankheit tritt meist herdweise auf und zeigt stets Neigung zur Gruppenbildung, die erstere ist diffus verbreitet; sie bevorzugt die Extremitäten, die Keratosis spinulosa den Rumpf. Bei dieser sind alle Efforeszenzen eines Herdes annähernd gleich groß, höchstens bis zur Größe eines Stecknadelkopfes; bei der Keratosis follicularis sind die Größendifferenzen zwischen den einzelnen Effloreszenzen ziemlich beträchtlich, von Stecknadelkopf- bis Hanfkorn-, ja bis Linsengröße. Den größten Unterschied bilden die Hornstachel, die bei der Keratosis spinulosa fein und dünn von heller Farbe gleichförmig gestaltet sind, während sie bei der Brookeschen Krankheit außerordentlich polymorph sind. Die einen übertreffen die des Lichen spinulosus um ein Vielfaches an Größe und Dicke, die anderen treten überhaupt nicht aus dem Follikel aus, sondern bilden die für die Affektion besonders charakteristischen komedoähnlichen Hornpfröpfe. Dunkle Färbung der Hornmassen, Pigmentierung der Effloreszenzen ist häufg bei Keratosis follicularis; in einzeInen Fällen ist auch stärkeres Hervortreten der Hautfelderung zwischen den Effloreszenzen beschrieben worden. Histologisch überwiegt bei der Keratosis follicularis die reine Hyperkeratose bei der Keratos spinulosa die Parakeratose. Daß nach alledem die Affektion des jungen Indiers zu der ersteren Krankheit zu rechnen ist, bedarf keiner weiteren Auseinandersetzung.

Etwas schwieriger als vom Lichen spinulosus gestaltet sich die Abtrennung der Keratosis follicularis Morrow - B rooke von der Darierschen Krankheit. Eine Verwirrung auf diesem Gebiete ist dadurch entstanden, da $\beta$ zwei amerikanische Autoren, White und Bowen, sichere Fälle von Dariers Dermatose sie selbst identifizieren ihre Fälle mit dieser - unter dem Namen "Keratosis follicularis" beschrieben haben. Dadurch ist 
bei vielen die Ansicht entstanden, daß die Morrow-Brookesche Keratosis follicularis überhaupt kein selbständiges Krankheitsbild darstelle, sondern zur Darierschen Krankheit gehöre. Daß dies nicht zutrifft, dafür scheint mir gerade unser Fall wieder einen neuen Beweis zu liefern. Schon die Lokalisation war der bei Darierscher Krankheit geradezu entgegengesetzt. Die Prädilektionstellen dieser Affektion waren bei unserem Patienten völlig frei: Inguinalgegend, Genitalien, Bauch, behaarter Kopf. Es fanden sich typische Hornstachel von fester trockener Konsistenz; fettige, borkige Massen fehiten vollkommen, ebenso jede Neigung zu Wucherungen. Noch stärker war die Differenz im histologischen Bild. Davon konnte ich mich besonders durch Vergleich mit Präparaten von zwei typischen Fällen Darierscher Krankheit überzeugen. Das eine stammt von dem kürzlich durch Bizoz zero publizierten Fall aus der Berner Klinik; ich verdanke es der Freundlichkeit von Herrn Prof. Jadassohn. Das andere von einem Herxbeimerschen Fall stellte Herr Reinhart mir liebenswürdigerweise zur Verfügung. Es fehlten in meinem Falle vor allem jene degenerativen Erscheinungen im Rete, die als Lückenbildung bei Darierscher Krankheit auftreten, worauf gerade Bizozzero wieder besonders die Aufmerksamkeit gelenkt hat. Es waren keine gewucherten Retezapfen, keine verlängerten Papillen zu sehen, es fehlte jede größere Einsenkung der Hornschicht außerhalb der Follikel. Die Kutis-Epithelgrenze verlief, von den durch Dilatation difformierten Follikeln abgesehen, durchaus normal.

Ein paar Worte wären noch über die "Corps ronds" und "Grains" zu sagen. Daß diese Gebilde nichts für die D a i r er sche Krankheit Charakteristisches sind, sondern bei allen möglichen mit unregelmäßiger Verhornung einhergehenden Prozessen vorkommen, ist seit langem bekannt. Darier selbst bildet sie in der "Pratique dermatologique" beim Epitheliom und bei Pagetscher Krankheit $a b$ und bezeichnet sie einfach als dyskeratotische Zellen. Nicht einmal die Reichlichkeit ihres Auftretens scheint für die Diagnose der Darierschen Krankheit irgend welche Bedeutung $\mathrm{zu}$ haben. Caspary hat einen charakteristischen Fall dieser Affektion geschildert, bei dem es 
nur mit Mühe gelang jene Zellformen nachzuweisen. Doctor hat in zwei Fällen überhaupt keine gefunden. Ich glaube daher, die Tatsache, daß sie in meinem Fall reichlich vorhanden waren, nicht für die Diagnose "Dariersche Krankheit" und gegen "Keratosis follicularis" verwerten zu dürfen, da alle andern Faktoren für die letztere sprechen. Es ist allerdings, soweit ich die Literatur kenne, das erste Mal, daß die dyskeratotischen Zellen Dariers bei der Keratosis follicularis Morrow-Brooke beobachtet worden sind.

Die Fälle von echter Keratosis follicularis M o r r o w -B r o o k e sind auch heute noch recht spärlich in der Literatur. Wieviele von den alten Fällen von "Acné cornée" dahin gehören, läßt sich nicht bestimmt sagen. Bro o ke erwähnt $\mathrm{Caz}$ e nave, Guibout, Leloir und Vidal. Als denjenigen, der zuerst die Krankheit unter dem Namen Keratosis follicularis beschrieben hat, nennt er mit Recht Morrow. Freilich will dieser Autor das Wesen der Affektion in einer Erkrankung der Talgdrüsen sehen. Es ist nicht unmöglich, daß diese Ansicht auf einer irrtümlichen Deutung des histologischen Bildes beruht, denn nach der Beschreibung und den Abbildungen Morrows scheint es fast, daß er die sackartig erweiterten Follikelhälse für verhornte Talgdrüsen gehalten hat. Bemerkenswert ist die Leukokeratose der Zungen- und Wangenschleimhaut in seinem Falle.

Am bekanntesten ist die Brookesche Schilderung der Krankheit geworden. Er beobachtete sie bei mehreren Angehörigen zweier Familien und schloß daraus auf infektiöse Ätiologie und Kontagiosität.

Histologisch studierte $\mathrm{Un} \mathbf{n}$ a die Keratosis follicularis. Es folgen dann einige Krankenvorstellungen, von Sabolotsky und von Sokoloff in der Moskauer, von Saalfeld in der Berliner dermatologischen Gesellschaft. Erst in den letzten Jahren ist das Thema wieder gründlicher bearbeitet worden von Samberger und von Gutmann. Beide beschäftigen sich ausführlich mit der Histologie des Leidens. Im wesentlichen stimmen diese Untersuchungen mit den Angaben Brookes und Unnas überein, nur über einzelne Fragen herrscht noch Uneinigkeit, so uiber die Beteiligung der Hornschicht außerhalb der Follikel und darüber, ob die Hyper- 
keratose durch erhöhte Produktion oder nur durch festeres Zusammenbalten der Hornmassen entsteht. Auch meine Befunde schließen sich dem gut an; nur das Vorkommen von "Corps ronds" und "Grains" ist neu. Es wäre hier schließlich noch zu diskutieren, ob nicht auch der zwei Geschwister betreffende Fall, der von Jadassohn und mir in der Ikonographia dermatologica beschrieben wurde, und der diesem fast kongruente Fall, den Bettmann auf dem 10. Deutschen Dermatologenkongreß in Frankfurt zeigte, zur Keratosis follicularis Morrow-Brooke gehören.

Die follikuläre Einzeleffloreszenz des Berner Falles war makroskopisch mit der des Indiers identisch, histologisch bestanden allerdings kleine Differenzen, wie das Fehlen von "Corps ronds" in jenem die Vermehrung des Keratohyalins und die Aufhellung der Zellen. Gemeinsam sind ferner all diesen Fällen mit dem Fall Morrows die Leukokeratosen der Mundschleimhaut. Die eigentümlichen Veränderungen von Nägeln, Handflächen und Fußsohlen bestanden dagegen nur in dem Berner und dem Heidelberger Fall. Die Zugehörigkeit gerade dieser beiden Fälle zur Keratosis follicularis Morrow-Brooke zu erweisen wäre insofern wichtig, als damit für die Ätiologie des Leidens eine sichere Anschauung gewonnen wäre. Denn daß es sich in diesen Fällen um eine auf kongenitaler Anlage beruhende Verhornungsanomalie handelt, kann doch kaum bezweifelt werden. Dasselbe möchte ich auch für den Fall des jungen Indiers annehmen. Dagegen entstand die Krankheit in den Fällen ron Samberger und Gutmann erst im reiferen Alter und trug wie in den Fällen von Brooke mehr vorübergehenden Charakter. Das letztere ist allerdings nicht von großer Bedeutung, da alle Fälle große Schwankungen zeigen; in dem Berner Fall z. B. entstanden die follikulären Effloreszenzen erst während des Aufenthaltes in der Klinik und verschwanden zeitweise fast ganz. Es wäre denkar, daß das Wesen der Krankheit auf einer kongenitalen Anlage beruht, die unter umständen erst im späteren Leben auf irgendwelche uns noch unbekannte Veranlassungen hin zur Ausbildung gelangt. Für eine parasitäre Ätiologie sind bisher keine Beweise geliefert worden; es ist nie ein Mikroorganismus gefunden worden; das 
sporadische, höchst seltene Auftreten einzelner Fälle in den verschiedensten Ländern spricht nicht dafür, nicht mehr auch das Vorkommen bei mehreren Mitgliedern einer Familie. Das läßt sich genau so gut für ene ererbte Disposition ins Treffen führen. Jedenfalls ist es richtig das Beiwort "contagiosa", das Brooke der Affektion beilegte, fallen zu lassen, und bis wir ihre wahre Natur kennen, einen indifferenten Namen zu wählen, der auch dem ersten Autor, der sie beschrieb, gerecht wird, sie also als "Keratosis follicularis MorrowBrooke" zu bezeichnen.

\section{Literatur.}

1. Adamson. Lichen spinulosus. Brit. journ. of Derm. 1905.

2. Bettmann. Pachyonychia congenita. Verhandlg. d. X. Kongr. d. Deutsch. Derm. Ges. Frankfurt 1908. pag. 378.

2. Bizozzero. Über die Dariersehe Dermatose. Areh. t. Derm. 1908. Bd. XCIII.

4. Bowen. Keratosis follicularis etc. Journ. of cut. and gen.-urin. diseases. 1896. Vol. 14. p. 209.

5. Brooke. Keratosis follicularis contagiosa. Intern. Atl. seltener Hautkrankheiten. 1892. Heft. 7.

6. Caspary. Über einen Fall von Darierscher Krankheit. Arch. f. Derm. Festschrift f. Kaposi. 1901. pag. 199.

7. Crocker, R. Diseases of the Skin. Vol. I. p. 552-560.

8. Darier. La pratique dermatologique. Vol. II. p. 402-404, Vol. III. p. 633, Vol. IV. p. $148 \mathrm{ff}$.

9. Doctor. Über das Verhältnis der Darierschen Krankbeit zar Ichthyosis. Arch. f. Derm. 1898. Bd. XLVI.

10. Gutmann. Ein Beitrag zur Kenntnis ungewöhnlicher Keratosisformen. Arch. f. Derm. 1906. Bd. LXXX. p. 93.

11. Jadassohn u. Lewandowsky. Pachyonychia congenita etc. Ikonographia dermatologica. 1906. Fasz. I. p. 29.

12. Ja novsky. Hyperkeratosis. Mračeks Handbuch d. Hautkrankheiten. Bd. III. p. 62-79.

13. Lesseliers. Contribution à l'étude du lichen scrofulosorum. Ann. de Dermat. 1906. 
14. Lewandowsky. Über Lichen spinulosus. Arch. f. Dermat. 1905. Bd. LXXIII.

15. Morrow. Keratosis follicularis etc. Journ. of cut. and gen.urin. diseases. 1886. Vol. IV. p. 257.

16. Piccardi. Keratosis pilaris e Keratosis spinulosa. Tesi di libera docenza. Turin 1906.

17. Saalfeld. Keratosis follicularis. Berlin. derm. Ges. 1899.

18. S a bolotsky. Keratosis follicularis. Venerol.-derm. Ges. Moskau

1898. Ref. Monatsh. f. prakt. Derm. Bd. XXVIII. p. 32.

19. Salinier. Keratosis spinulosa. Thèse de Toulouse. 1906.

20. Samberger. Zur Pathologie der Hyperkeratosis. Archiv f. Derm. 1905. Bd. LXXVI. p. 241.

21. Sok ol off. Keratosis follicularis. Venerol. derm. Ges. Moskau 1901. Ref. Monatsh. f. prakt. Derm. Bd. XXXIII. p. 593.

22. Thibierge. La pratique dermatologique. Kap. Acné cornée. Bd. I. p. 251.

23. Unna. Histopathologie der Hautkrankheiten.

24. White. Keratosis follicularis. Journ, of cut. and gen.-urin. diseases 1889 и. 1890. 